\title{
La educación en des-tiempos del Covid-19. Crisis, tecnologías y futuro
}

Education in the dis-times of Covid-19. Crisis, technologies and the future

\author{
Rolando Barral Zegarra \\ rolyba17@hotmail.com \\ Código ORCID: 0000-0001-9934-4935 \\ Universidad Mayor de San Andrés, La Paz, Bolivia
}

RESUMEN

- Artículo recibido en octubre 2020

- Arbitrado en noviembre 2020

- Aceptado en diciembre 2020

- Publicado en enero 2021

El presente trabajo es una reflexión de los problemas que se presenta con la crisis de la Pandemia a causa del Covid-19, el rol de las tecnologías relacionados con la Educación Virtual y la Educación a Distancia y la prospectiva en la cual el sistema educativo se manifiesta por su inercia, anacronismo y retroceso en la solución de los problemas que presentan las sociedades a nivel nacional y regional.

Palabras clave: Educación; comunicación; crisis; tecnología; Covid-19

\section{ABSTRACT}

This work is a reflection of the problems that arises with the crisis of the Pandemic caused by Covid-19, the role of technologies related to Virtual Education and Distance Education and the prospects in which the educational system is manifested by its inertia, anachronism and setback in solving the problems that societies present at the national and regional level.

Keywords: Education; communication; crisis; technology; Covid-19 
"Cuando eres producto de tu cultura, sin cuestionarte nada, te conviertes en un robot". Anthony de Melo

"El verdadero peligro de la tecnológica época en que vivimos no es tanto que las máquinas lleguen a pensar como hombres, sino que los hombres den en pensar como máquinas". S. J. H.

\section{No educar para el futuro es un suicidio}

El futuro está aquí y nos sobrepasa. Se teorizaba que las pandemias iban a venir más adelante y llegó una, con un impacto global insólito. El Covid-19 está haciendo estragos en todas las dimensiones: la salud, la economía, la sociedad, la política y por supuesto la educación. La humanidad está en una situación de guerra, expresan algunos científicos y periodistas. No es un sueño, es la realidad.

De la noche a la mañana el aislamiento fue forzoso para atenuar el Covid-19. En ese contexto, como dice la gente: la necesidad es madre de todos los inventos. La educación formal cerró sus puertas. Esa escuela odiada y anunciada muerta ya no era más. Y se abrió un horizonte en el campo educativo mediado por la tecnología; sin embargo, no todos estaban preparados. Las crisis siempre ponen de manifiesto las desigualdades sociales y educativas. En nuestro medio, el sistema educativo precario, anacrónico, una vez más, mostró su cuerpo anquilosado y burocrático.

Desde el poder central y la sociedad se elucubra salidas a la crisis: educación a distancia, virtual, transmedia, etc. En concreto, no estamos preparados para lo inesperado, el sistema educativo no está a la altura de los acontecimientos trágicos porque la educación estuvo invariablemente aferrada al pasado. Se reacciona como amebas frente al estímulo. Se es reactivo pero no proactivo. Se hace diagnosis pero no prognosis, se vive en la tradición pero no se hace prospectiva. Por ello, el sistema educativo "deforma" o "deseduca" para en el día anterior y no educa para el futuro.

Un escritor, Ortega y Gasset advirtió a los educadores en estos términos: "Los pedagogos siempre llegan tarde a los descubrimientos de las ciencias". Siguiendo la lógica crítica, gran parte de los políticos no ven más allá de sus narices, como los burócratas -frente a los problemas- se encargan de hacer Decretos y Reglamentos (como el de "Teletrabajo") que ni los cumplen, en vez de buscar y formular políticas públicas con participación social. 
Toda crisis anuncia una nueva etapa. Los investigadores no deben dejar pasar la oportunidad para seguir descubriendo en estos nuevos espacios, más aún, cuando la educación apuesta por la ciencia y la tecnología, como no había pasado antes. Es decir, es tiempo oportuno para que los países pobres inviertan en investigación y así empezar a dejar de serlo, poco a poco. Edmund Carpenter había lanzado una observación: "Los nuevos medios -el cine, la radio, la televisión- crean nuevos lenguajes y su gramática es todavía desconocida (1974, p. 213). Cada uno de ellos codifica la realidad de modo diferente; cada uno de ellos oculta una metafísica única". En la actualidad, el ordenador, la cibernética, la informática, el internet, el celular inteligente, la tecnología digital, la realidad virtual, la inteligencia artificial, son muestras evidentes de la evolución del átomo al bit y el cubit (esta última es la unidad básica de información de la computación cuántica). De otra forma, lo dijo Bacon: "el conocimiento es poder", la información es poder, la tecnología es poder. Esta crisis, ha demostrado que hay una zona $u$ horizonte de demanda educativa y tecnológica, en conceptos del enfoque sociocultural es una "zona de desarrollo próximo": espacio donde la investigación es una necesidad capital para un futuro de incertidumbres. Por tanto, es un imperativo formar futurólogos.
Además de estudiar nuevos lenguajes creados, la gramática de las nuevas tecnologías, es necesario estudiar su historia y sus genealogías, sus vínculos con el poder, sus intenciones político-pedagógicas y las geopolíticas del conocimiento. Asimismo, indagar el mapa cognitivo que diseñan en la mente de los usuarios, sus verdaderos propósitos; de otra forma, la semiótica de los nuevos lenguajes de la tecnología.

¿Qué se les puede pedir a los gobernantes y ministros de educación que vienen de la cultura de la máquina de escribir? En el contexto internacional, los gobernantes que subestimaron y se mofaron del Covid-19, me refiero a Donald Trump de EEUU, Andrés Manuel López Obrador de México, Jair Bolsonaro de Brasil, entre otros, los datos y las estadísticas del colapso en la salud, son un revés para sus juicios apresurados, porque hicieron lecturas economicistas e ideológicas y no científicas del problema del Covid-19. Los poderes mundiales toman decisiones en diferentes dimensiones a nivel planetario. No sabemos con precisión cuáles son esas medidas, pero sufrimos las consecuencias como es el caso del Covid-19 y el problema: saludenfermedad que atraviesa el mundo de hoy.

Volviendo al tema educativo, otra advertencia de un visionario como MacLuhan, sirve para la reflexión y la 
polémica: "La pedagogía contemporánea no corresponde a la era de la electricidad; se quedó en la era de la escritura" (citado por Porcher, 1976, p. 32). Queda el desafío de pedagogizar la tecnología y digitalizar la misma, fundamentalmente humanizar la tecnología ante los inminentes riesgos que esto supone, para enfrentar con más eficacia los problemas que ha puesto de manifiesto el Covid-19. También es muy importante didactizar el proceso de enseñanza-aprendizaje usando los recursos tecnológicos de la información. En fin son nuevas competencias que los docentes deben aprender.

\section{Educaciones y tecnologías}

Es una exigencia en la situación actual diversificar el concepto de educación en un mundo plural y abierto -varios educadores ya lo hicieron-, y relacionar las educaciones a las tecnologías que emergen en nuestro mundo hoy amenazado.

Tecnología educativa. En el desarrollo de la educación en general la tecnología educativa surgió como un designio manipulador porque estaba sustentado por la corriente psicológica conductista; no era su propósito fundarse en la psicología cognitiva y menos apoyarse en la corriente sociocultural. Pero el desarrollo posterior exigía que el sujeto piense y no sólo actúe. El progreso de la ciencia y la tecnología requiere sujetos creativos lo cual motivó el desplazamiento del modelo conductual.
Ligado al pensamiento, hoy por hoy, los sistemas sociales exigen que el sujeto desarrolle los afectos y las emociones, ignorados por la tecnología educativa tradicional.

La historia da cuenta que la incorporación de la imprenta en la escuela con Celestin Freinet, logró una revolución cognitiva. Consecutivamente, la inserción de la computadora en la escuela, logró una revolución en el aprendizaje. Hoy la información está a la mano del usuario o del estudiante de cualquier nivel educativo. Sin embargo, la tecnología educativa no es una panacea. Los estudios sobre esta relación, dan cuenta que no es suficiente dotar ordenadores, se debe crear condiciones para que funcione, por tanto, se requiere toda una infraestructura. Un ejemplo: dotar a los alumnos computadoras y no dar el servicio de internet gratuito, es lo mismo que nada; peor aún, si se tiene un satélite artificial que no facilita el servicio de internet con la velocidad deseable y que se pague: es un engaño.

Escuela paralela. En la década de los 70 del anterior siglo, se criticó y se problematizó la función de la escuela y aparecieron varias teorías como el de la "desescolarización" (Iván Illich, 1970). Otros autores vaticinaron su muerte (Everett Reimer, 1971), y no ocultaron su odio tenaz (Emile Chanel, 1976). Sin embargo, otra propuesta interesante fue la "La escuela paralela" (Louis Porcher, 1976). La escuela paralela llegaba a los 
alumnos y a los que no lo eran con información, conocimientos y formación cultural. Sus instrumentos fueron los medios de comunicación de masas: la prensa, la radio, el cine y la televisión. Una de sus críticas a las reformas educativas y al sistema tradicional fue punzante: "Las reformas pasan, el sistema queda" (Tardi, citado por Porcher, 1976, p. 16). Claro está, se empezó a valorar la incidencia de los medios en la cultura y la educación a través de la comunicación. Se apreció el valor pedagógico de los medios mencionados.

Sin embargo, la comunicación y la educación hay que comprenderla, estudiarla y problematizarla en su contradicción: "incomunicación" y "deseducación". Diversas investigaciones revelan los prejuicios y estereotipos que se producen y circulan por esos medios. No en vano Karl Popper tituló uno de sus trabajos: La televisión es mala maestra (2002). Y en nuestro continente Juan Díaz Bordenave reflexionó al respecto: "Toda educación es comunicación, pero no toda comunicación es educativa". Lo anterior se convirtió en todo un principio pedagógico desde la experiencia latinoamericana, lo que se denomina hoy como la "Educomunicación".

Informática y educación. En este nuevo escenario, un nuevo sujeto surgió como producto del avance de las nuevas tecnologías: el homo informaticus. Este ser individual y colectivo es el nuevo consumidor de información, pero también un proveedor de datos iun tonto útil? ¿Es para hacerlo más dependiente y manipulable?

David Hawkridge en su obra: Informática y educación, establece ideas muy significativas -que abreviamospara reconfigurar esta temática: La nueva tecnología de la información se basa en tres tecnologías complejas que han confluido: la computación, la microelectrónica y las telecomunicaciones. Los educandos tienen la oportunidad de aprender en más de una modalidad. Los problemas educativos que se plantean con frecuencia son: 1.- La educación depende del software. 2.- El aprendizaje mediatizado en oposición al aprendizaje personal. 3.- Los docentes no podrán ni querrán realizar cambios de roles. 4.La tecnología acrecentará el elitismo educacional. (Otro grupo de crítica considera que la nueva tecnología de la información será barata $\mathrm{y}$, en consecuencia, acrecentará las oportunidades de aprendizaje). 5.- Los sistemas educacionales formales se verán debilitados. Así, resume: "La tecnología dominará la educación". En la indagación el autor citado, a su vez rescata la posición pesimista: "habrá instituciones educativas en que se usará tecnología de la información de una manera deshumanizante" (1985, pp. 16200)

Las tecnologías de la información están revolucionando al mundo y simultáneamente está cambiando la 
mentalidad del ser humano. La informática y la educación son problemas que se debaten en diferentes espacios públicos y privados, unos a favor y otros en contra, tratando de interpretar el futuro. En otras palabras, la tecnología aplicada a la educación promueve una educación abierta a nuevas formas y modos de aprendizaje y de enseñanza. No sólo se observa problemas, sino también soluciones. Como todo tiene su contrario, la tecnología y la informática mal usadas están deshumanizando.

Alfabetización informática. El escenario de crisis que ha traído el Covid-19 obligó a los gobernantes decretar el aislamiento social para cuidar la salud, y se suspendieron las clases presenciales escolares en el campo de la educación formal y no formal, esta situación ha puesto de manifiesto el "analfabetismo tecnológico" de autoridades, docentes y alumnos en diferente medida. La interrupción de clases fue sorpresiva y la falta de capacitación docente corroboró esta realidad. Los docentes que trabajan en la educación a distancia a nivel nacional e internacional no tuvieron problemas en adaptarse a las clases virtuales, la mayoría no. Sin embargo, no es su culpa, es responsabilidad del sistema educativo que no los capacitó y menos preparó para escenarios como el que estamos sobrellevando.

En un anterior texto mío sobre: Más analfabetismos (2009), me referí al "analfabetismo tecnológico" y describí que muchos hijos que tienen acceso a la tecnología digital enseñan a sus padres sobre el manejo de ciertas aplicaciones en el ordenador y los "celulares inteligentes". Por otra parte, se percibe que numerosos estudiantes saben más de aplicaciones tecnológicas que sus profesores, claro está, con excepciones.

Algunos indicadores de este "analfabetismo tecnológico" incluido de los que gobiernan, es que se dotaron en el país computadoras a docentes y discentes hace más de diez años sin tomar en cuenta que todo equipo tecnológico con el tiempo se vuelve obsoleto. En muchas unidades educativas guardaron esos equipos y no se distribuyeron a los alumnos, menos se crearon las condiciones para su uso, como hubiera sido conveniente el implantar el acceso gratuito a internet; así se concretó en el vecino país de Uruguay (Plan Ceibal 2007): este nación es todo un ejemplo de no hacer demagogia con las dotaciones de equipos tecnológicos a su población estudiantil. Queda como desafío para el Ministerio de Educación, la sociedad y sus instituciones educativas $\mathrm{y}$ universitarias: disminuir la "brecha digital": como expresan los entendidos, la misma es una nueva forma de exclusión social y se debe buscar una mayor democratización al respecto.

Educación a distancia. $\mathrm{La}$ diversificación del proceso educativo, toma en cuenta a los sujetos, sus áreas de desarrollo, los ejes temáticos 
transversales, también las modalidades de atención presencial y no presencial como es la educación a distancia. Se ha definido la misma como un "modo de enseñanza que no involucre la presencia física del maestro encargado de impartirla en el lugar en que se la recibe o en el cual el maestro sólo se halla presente en ciertos momentos para tareas específicas. La comunicación docente-educando se establece en particular mediante la correspondencia, los impresos, los diversos medios de difusión audiovisuales, la informática y ciertas agrupaciones de alumnos" UNESCO. (1988, p. 87)

La educación a distancia concibe el proceso de enseñanza-aprendizaje que tiene su centro en el alumno. Es un proceso de auto-instrucción, apoyado con materiales pedagógicamente elaborados que exigen un estudio individual y responsable, al igual que una autoevaluación permanente. (ICFES). En este proceso de autoformación asistida, el participante es el centro en su contexto cultural específico, porque se parte de sus necesidades, sus intereses y su rol en la comunidad. Uno de los problemas, entre otros, que presenta la educación a distancia y virtual son los altos índices de "abandono", los participantes por diferentes causas y motivos desisten de los programas académicos después que los inician.

Educación virtual. El proceso de enseñanza-aprendizaje vía internet es lo que se ha llamado "educación virtual".
Esta modalidad es otra opción planificada por diferentes sistemas educativos en varios países, una de esas iniciativas es lo que se ha llamado: "Escuela en casa", esta experiencia se aplica desde el preescolar hasta niveles superiores: los sujetos deben rendir exámenes con base a estándares a nivel nacional e internacional que los sistemas educativos organizan.

En este periodo especial del Covid19, las oportunidades de la "educación virtual" se han abierto con todas las ventajas y desventajas propias de la coyuntura. Una nueva nomenclatura ya es familiar en los docentes, aunque otros ya estuvieron habituados mucho más antes por su vinculación con el mundo laboral tecnológico, esos sistemas son: plataformas digitales, Software educativo, entorno digital, etc. Y también aplicaciones como el Zoom, Google Classroom, Discord, Gotmeeting, etc. Una ventaja es que una persona puede instalar aplicaciones de software con tutoriales prediseñados, de ahí su carácter auto-instructivo.

Tecnólogos y educadores se preguntan ¿Hay algún paradigma pedagógico detrás de tan sofisticada tecnología? ¡Claro que sí! Antes está la dominación del Capital tecnológicofinanciero. $\mathrm{Y}$ el modelo educativo de "contenidos" mediado por la tecnología está orientado a diseñar un mapa cognitivo funcional, eficiente $\mathrm{y}$ dependiente. Una de sus ventajas es que 
transmite aspectos conceptuales, información útil (saber), y sus desventajas tendría dificultades en el aspecto procedimental (saber hacer) y enormes vacíos en lo actitudinal, los valores y los afectos (Saber ser). Empero, en la dimensión procedimental hay experiencias de operaciones de telecirugía asistida con imágenes 3D. Por supuesto que la educación integral no es considerada por la educación fragmentaria: funcional-unilateral pero no íntegra.

Este tipo de educación tecnológica del siglo XXI ya se había planeado hace mucho tiempo atrás con el uso masivo del internet, el audio y el video, las aplicaciones cada vez más modernizadas, la fibra óptica con más ancho de banda y la multidifusión de los datos a través de los ordenadores y celulares inteligentes, etc. Querer ignorar esta realidad y evadirla es retroceder, lo que se debe hacer es asimilar y superar (aprender y proyectar) y ponerla al servicio del bienestar humano y no a la inversa, en otras palabras, del Capital tecnológico y los centros de poder dominantes. Una orientación simple es dirigirla a la lucha contra la pobreza y la desigualdad social.

Inteligencia Artificial. Otro de los horizontes abiertos a la educación es la inteligencia artificial (IA). Lasse Rouhiainen en su texto: Inteligencia artificial, define la misma como "la habilidad de los ordenadores para hacer actividades que normalmente requieren inteligencia humana". Asimismo, agrega: "la IA es la capacidad de las máquinas para usar algoritmos, aprender de los datos y utilizar lo aprendido en la toma de decisiones tal y como lo haría un ser humano" (2019, pp. 14-15). Ahora, analizamos y contrastamos de forma precisa algunas ideas centrales relacionadas de la IA con la educación.

El consultor arriba mencionado, relaciona el impacto de la IA en diferentes espacios de la vida y de la sociedad. En ese sentido, arguye sobre la IA y la educación: la inteligencia artificial tendría el potencial de crear sistemas educativos personalizados y eficaces. Frente a los sistemas educativos ineficientes y burocráticos la IA sería una buena alternativa. La IA está cambiando el mercado laboral de forma significativa, las personas serán reeducadas profesionalmente. Lo anterior, se funda en un proceso vital como es la "reeducación" y que está vinculado con la educación permanente y la capacitación pensando en los empleos y las profesiones del futuro.

Otra demostración importante del autor en cuestión es, que las tecnologías emergentes cambiarán la educación para siempre y mejorarían los sistemas educativos en todo el mundo. En esa perspectiva, el sistema educativo en sus diferentes niveles: primaria, secundaria y superior, serían beneficiados con ejes temáticos ignorados por el sistema educativo y la escuela tradicional aún 
vigentes. Estos, serían: inteligencia emocional, social, ambiental, también la creatividad y el desarrollo personal, entre otros. El autor destaca los cursos $e$ learning; además, muestra modelos como ser: las plataformas de aprendizaje personalizados, los tutores de inteligencia artificial, los juegos personalizados y también rescata un principio pedagógico el cual establece "que los juegos son una de las mejores formas de aprender algo nuevo" (2019, pp. 32-60). En síntesis, el aporte de la IA en el campo de la educación es inexorable $y$ en la investigación científica -como ya sucede- es una herramienta muy útil.

\section{Sistema educativo $\mathrm{y} / \mathrm{e}$ in-competencias docentes}

Las sociedades en el mundo entero han criticado de forma mordaz a los sistemas educativos, a la escuela y sus agentes. Lo anterior se reflejó en teorías pedagógicas, graffitis, imágenes, canciones como el de Pink Floyd "Un ladrillo en la pared", pero el sistema sigue inalterable. Este des-tiempo de crisis del Covid-19 ha desnudado una vez más que los sistemas educativos son caducos. El mundo productivo, laboral y tecnológico son dinámicos, los sistemas educativos ¡no! ¿Por qué?

"La lentitud con que son incorporadas las innovaciones a los sistemas educativos... la administración de la educación parece continuar navegando dentro de la etapa artesanal... La ineficacia de la administración de los sistemas educativos parece reposar... en el hecho de que los Ministerios de Educación han ido reformándose en base agregados y yuxtaposición de nuevas estructuras, pero no en función de verdadera vertebración que les permita atender las necesidades presentes y futuras". La anterior radiografía fue escrita por Ruth Lerner en el año 1972; con todo, parece que habría escrito hoy. ¿Por cuánto tiempo más el sistema educativo seguirá desfasado? Un dato de lo anterior, es que no tenemos docentes capacitados para la educación a distancia y virtual, en cantidad y calidad suficientes para afrontar el desafío, después del hecho, posterior al Decreto de la cuarentena recién se piensa implementar la formación tecnológica para la educación virtual. Y los docentes que utilizan las plataformas digitales, dan un "salto al vacío". Y eso pasa en todos niveles incluido la educación universitaria: reproducen las clases tradicionales, es decir: nuevos medios, viejas enseñanzas; nuevos medios, viejos aprendizajes. Con excepción de maestros y maestras que están vinculados a la educación y la tecnología. Es más, en nuestro medio docentes de base, propusieron iniciativas de educación a distancia y virtual al Ministerio de Educación. Pero para la burocracia toda iniciativa es un insulto.

Con relación a las competencias docentes para estos tiempos de crisis, se puede escribir todo un tratado; sin embargo, la brevedad también es una 
cualidad, por lo tanto, advertimos con Willam A. Ward: "El profesor mediocre dice. El buen profesor explica. E1 profesor superior demuestra. El gran profesor inspira".

Esa inspiración -entre otras idoneidades- se interpreta: inicialmente los educadores deben ser personas que amen su profesión, tengan reflexión contextual y criterio científico, además desarrollen pensamiento crítico $\mathrm{y}$ creativo, demuestren ser sujetos con visión de futuro (proactivos), y manejen tecnologías de información; o sea el docente como guía, organizador $\mathrm{y}$ mediador de la cultura.

Con relación a los docentes, los niños y estos medios: una cierta "tecnofobia" caracteriza a los profesores, y mucha familiaridad de los niños con los ordenadores. Bill Gates ya había advertido en su país y lo expresó en su libro: Camino al futuro: "A menudo, los profesores no saben exactamente qué hacer con las computadoras...". "Las computadoras ponen nerviosas a todas las personas antes de llegar a entenderlas, menos a los niños". "Los niños y las computadoras se llevan muy bien..." (1997, pp. 200-277). No obstante, los escenarios están cambiando y los procesos también requieren un cambio de mentalidad y de actitud. Muchos educadores y educadoras ya empezaron antes de esta pandemia, porque el ser humano tiene la capacidad de proyectarse al futuro. Es decir, no anclarse en el pasado, sino ser visionarios de vanguardia.

\section{CONCLUSIONES}

La crisis de la salud ha puesto de manifiesto que las tecnologías y la educación virtual y a distancia pueden contribuir en la continuidad del proceso educativo; sin embargo, las desigualdades socioeconómicas se manifiestan también en los déficits de acceso a las tecnologías y a la conectividad abierta al internet. Por todo lo anterior, el futuro queda postergado por un sistema educativo que no está a la altura de los grandes desafíos sociales y las problemáticas globales y locales.

\section{REFERENCIAS}

Barral, Rolando. "E1 Diario" Más analfabetismos 11-13-16 de marzo, La Paz, 2009

Carpenter, Edmund; McLujan, Marshall (1974). El aula sin muros. Barcelona: LAIA

Gates, Bill (1997). Camino al futuro. Madrid: McGraw-Hill

Hawkridge, David (1985). Informática y educación. Buenos Aires: Kapelusz

McLuhan, Marshall (2015). Inédito. Buenos Aires: La marca

Popper, Karl R. (2002). La televisión es mala maestra. México: Fondo de cultura económica

Rouhiainen, Lasse (2019). Inteligencia artificial.101 cosas que debes saber hoy sobre nuestro futuro Barcelona: Alienta

UNESCO (1994). ¿Qué formación para los maestros? París: Organización de las Naciones Unidas para la Educación, la Ciencia y la Cultura 